DW. ALS in Rochester, Minnesota, 1925-1977. Neurology 1980;30:463-70.

4 Yoshida S, Mulder DW, Kurland LT, Chu C-P, Okazaki H. Follow-up study on amyotrophic lateral sclerosis in Rochester, Minnesota, 1925 through 1984. Neuroepidemiology, (in press).

5 Kurland LT, Choi NW, Sayre GP. Implications of incidence and geographic patterns on the classification of amyotrophic lateral sclerosis. In: Norris FH Jr, Kurland LT, eds. Motor Neuron Diseases. New York: Grune \& Stratton, 1969:28-50.

\section{Li et al reply}

We were interested to see Drs Kurland and Mulder's comments on age specific incidence rates in motor neuron disease, and note that our findings are in agreement with theirs. Clearly, this implies a common causative factor operating in these widely separated environments and it would be interesting to compare cohort data for these different populations. We agree with Kurland and Mulder that the diagnosis of motor neuron disease is probably often overlooked in elderly patients, thus resulting in an under-estimate of incidence rates in older populations. This is a common limitation of epidemiological data taken from retrospective surveys of case-notes, but in our work we have tried to exclude this factor as far as possible by utilising data from two separate Health Authorities, both with relatively well-organised neurological services. Similar trends were found in the two sets of data.

\section{Cryptococcal meningitis and cerebral toxo- plasmosis in a patient with acquired immunodeficiency syndrome.}

Sir: We were very interested in the short report of Bahls and Sumi ${ }^{1}$ about the documented simultaneous infection of the central nervous system with Toxoplasma gondii and Cryptococcus neoformans in an AIDS patient. We have also observed such an association on the same underlying disease.

A 26 year old homosexual man was admitted with fever, cough and meningitis. Transbronchial biopsy, blood and CSF cultures showed Cryptococcus neoformans. CD4/CD8 lymphocytes ratio was under $0 \cdot 2$. Serum sample was LAV/HTLV III antibody positive by two different techniques (ELISA, Western blott). Despite amphotericin B and 5-fluorocytosine in combination, seizures and confusion with right hemiparesis appeared. The cranial computed tomographic scan revealed three ring-enhancing mass lesions. Echoguided neurosurgical puncture of the left parietal mass lesion allowed brain biopsy which showed Cryptococcus neoformans (PAS stain). The patient died 4 weeks later. Toxoplasmosis serological and CSF tests were non-diagnostic. Brain culture from mice after intraperitoneal inoculation were positive for Toxoplasma gondii.

This new case emphasises the possibility of an infectious agent hiding another. Brain biopsy is indicated in such patients because of the lack of correlation between clinical presentation, CT scan appearance of mass lesions in the central nervous system, isolation of an infectious agent anywhere and the specific diagnosis of mass lesions. ${ }^{2}$ In this case, however, despite a brain biopsy in an affected area, it was not very useful, because routine haematoxylin and zosin stain was negative for $\boldsymbol{T}$ gondii, and culture from laboratory animal was necessarily slow. The immunohistological staining with peroxydase anti-peroxydase stain methodfast and specific diagnosis procedure for $T$ gondii $^{3}$ must be recommended, particularly in immunodeficient patients needing a rapid and specific diagnosis, critical to the directing of appropriate and urgent therapy for a potentially curable condition.

F LUCHT

$J$ JAUBERT

J HUPPERT

R TRAN MAHN SUNG

JC LEPETIT

H ROUSSET

Hospital de Belleune, Chru de St Etienne, Blvd Pasteur, 42023 St Etienne Cedex, France.

\section{References}

${ }^{1}$ Bahls F, Sumi SM. Cryptococcal meningitis and cerebral toxoplasmosis in a patient with acquired immunodeficiency syndrome. $J$ Neurol Neurosurg Psychiatry 1986;49: 328-30.

${ }^{2}$ Levy RM, Pons VG, Rosenblum ML. Intracerebral mass lesions in the acquired immunodeficiency syndrome. $N$ Engl $J$ Med 1983;309:1454-5.

${ }^{3}$ Conley FK, Jenkins KA, Remington JS. Toxoplasma gondii infection of the central nervous system: use of the peroxydase antiperoxydase method to demonstrate Toxoplasma in formalin-fixed, paraffin embedded tissue specimen. Hum Pathol 1981;12:690-8
Cryptococcal meningitis and cerebral toxo- plasmosis in AIDS: another case report.

Sir: We read with interest the Short report of Bahls and Sumi $^{1}$ on the association of cryptococcal meningitis and cerebral toxoplasmosis in a patient with Acquired Immune $T$ Deficiency Syndrome (AIDS) and we would like to document a further case.

A 34 year old homosexual male was $\stackrel{0}{\Rightarrow}$ admitted with a 4 months history of slight fever, cough with occasional haemoptysis $\vec{\Rightarrow}$. and weight loss. Three months later he devel- $\stackrel{\oplus}{+}$ oped a headache. One day before admission $\bar{C}$ the patient became less alert. Neurological $\overline{0}$ examination showed a comatose patient $\frac{\bar{D}}{D}$ with nuchal rigidity and left facial paralysis. $\mathbb{D}$ The diagnosis of AIDS and cryptococcal meningitis was made by clinical and labora- $\Leftarrow$ tory evaluation and was treated with ampho- $\overrightarrow{0}$ tericine $B$ and 5-fluorocytosine. After 2 months he developed progressive hemi- $\vec{\omega}$ paresis and CT scan showed multiple, ring- $D$ enhancing, low-density lesions. Slightly increased serologic titres against Toxo- 0 plasma gondii were detected and therapy of with pyrimetamine and sulfonamides was consequently started. In the next week the patient quickly improved, the CT lesion $\vec{\sigma}$ disappeared and he soon became asympo 을 tomatic. Unfortunately, after one and a hag months, therapy was discontinued becauso severe pancytopenia had developed, and he progressively experienced again right limi weakness and aphasia. Later, he died pneumonitis from Pneumocystis carin Pathological findings in the central nervous system revealed cryptococcal meningoencephalitis, multiple necrotising abscesses for Toxoplasma gondii and medulla and spinal cord infection by cytomegalovirus.

In our opinion, taking into account that cryptococcus and toxoplasma are both common causes of infection in patients with AIDS, ${ }^{2-5}$ their association may frequently occur. Consequently, we suggest the immediate onset of therapy with pyrimethamine and sulfadiazines if the diagnosis of toxoplasmosis is suspected ${ }^{5}$ in patients with cryptococcal meningitis and AIDS. Brain biopsy should be reserved for cases with poor response to treatment because its use is limited by its potential morbility and the presence of false-negative results.

I BONAVENTURA IBARS J MATIAS-GUIU C CERVERA A CODINA PUIGGROS Neurology Service of the Hospital del Valle de Hebrón. Universidad Autónoma de Barcelona

Spain 